\title{
Correlation strength, Lifshitz transition and the emergence of a two- to three-dimensional crossover in FeSe under pressure
}

\author{
S. L. Skornyakov, ${ }^{1,2}$ V. I. Anisimov,,$^{1,2}$ D. Vollhardt ${ }^{3}$ and I. Leonov ${ }^{1,4}$ \\ ${ }^{1}$ Institute of Metal Physics, Sofia Kovalevskaya Street 18, 620219 Yekaterinburg GSP-170, Russia \\ ${ }^{2}$ Ural Federal University, 620002 Yekaterinburg, Russia \\ ${ }^{3}$ Theoretical Physics III, Center for Electronic Correlations and Magnetism, \\ Institute of Physics, University of Augsburg, 86135 Augsburg, Germany \\ ${ }^{4}$ Materials Modeling and Development Laboratory, \\ National University of Science and Technology 'MISIS', 119049 Moscow, Russia
}

(Dated: October 13, 2018)

\begin{abstract}
We report a detailed theoretical study of the electronic structure, spectral properties, and lattice parameters of bulk FeSe under pressure using a fully charge self-consistent implementation of the density functional theory plus dynamical mean-field theory method (DFT+DMFT). In particular, we perform a structural optimization and compute the evolution of the lattice parameters (volume, $c / a$ ratio, and the internal $z$ position of Se) and the electronic structure of the tetragonal (space group $P 4 / \mathrm{nmm}$ ) unit cell of paramagnetic FeSe. Our results for the lattice parameters obtained by structural optimization of FeSe using DFT+DMFT are in good quantitative agreement with experiment. The $c / a$ ratio is slightly overestimated by about $3 \%$, presumably due to the absence of the van der Waals interactions between the FeSe layers in our calculations. The lattice parameters determined within nonmagnetic DFT are off the experimental values by a remarkable $\sim 6-15 \%$, implying a crucial importance of electron correlations in determining the correct lattice properties of FeSe. Upon compression to $10 \mathrm{GPa}$, the $c / a$ ratio and the lattice volume show a linear-like decrease by 2 and $10 \%$, respectively, while the Se $z$ coordinate weakly increases by $\sim 2 \%$. Most importantly, our results reveal a topological change of the Fermi surface (Lifshitz transition) which is accompanied by a two- to three-dimensional crossover. Our results indicate a small reduction of the quasiparticle mass renormalization $\mathrm{m}^{*} / \mathrm{m}$ by about $5 \%$ for the $e$ and less than $1 \%$ for the $t_{2}$ states, as compared to ambient pressure. The behavior of the momentum-resolved magnetic susceptibility $\chi(\mathbf{q})$ shows no topological changes of magnetic correlations under pressure, but demonstrates a reduction of the degree of the in-plane $(\pi, \pi)$ stripe-type nesting. Our results for the electronic structure and lattice parameters of $\mathrm{FeSe}$ are in good qualitative agreement with recent experiments on its isoelectronic counterpart $\mathrm{FeSe}_{1-x} \mathrm{~S}$.
\end{abstract}

PACS numbers: 71.27.+a, 71.10.-w, 79.60.-i

\section{INTRODUCTION}

Iron-chalcogenides $(\mathrm{FeCh})$ with the $\mathrm{PbO}$ crystal structure have attracted much attention recently due to their anomalous properties caused by the complex interplay between electronic, magnetic, and lattice degrees of freedom [1]. In this context, FeSe is one of the most wellknown members of the so-called 11-family, which shows remarkable connections between magnetism, nematicity, and superconductivity. FeSe has the same planar structure of the $\mathrm{FeCh}$ tetrahedra planes as the iron pnictides, bound together by the van der Waals interaction, but without separating layers [2, 3]. In fact, it is structurally the simplest among other Fe-based superconductors, with a superconducting phase emerging below a critical temperature $T_{c} \sim 8 \mathrm{~K}$ without applying doping and/or external pressure [4]. In contrast to iron pnictides, FeSe shows no long-range magnetic order, but displays a strong enhancement of short-range spin fluctuations near $T_{c}$, with a resonance at the $(\pi, \pi)$ magnetic vector (stripe-type) in the spin excitation spectra [5]. Moreover, similar to many other Fe-based materials, it exhibits a tetragonal-to-orthorhombic transition to a nematic phase below $T_{s} \sim 90 \mathrm{~K}$, with a spontaneously broken lattice rotational $C_{4}$ symmetry in the $a b$ plane $[1,6,6]$.

Upon compression, $T_{s}$ appears to decrease, followed by the emergence of a magnetically long-range ordered phase at $\sim 1 \mathrm{GPa}$ [8]. It leads to a dramatic increase of the critical temperature $T_{c}$ to a maximum of about $37 \mathrm{~K}$ at $\sim 6 \mathrm{GPa}$, implying that $T_{c}$ depends very sensitively on even a moderate change of the lattice volume [5, 9]. Furthermore, it has been recently shown that the electronic and lattice properties of bulk FeSe can be effectively tuned by the isoelectronic substitution of Se by $\mathrm{S}$ and Te, respectively. Namely, $\mathrm{S}$ has a smaller ionic radius than Se and hence results in a compression of the unit cell, while Te has a larger ionic radius, leading to an expansion of the lattice. In the expanded lattice $T_{c}$ is found to increase up to $14 \mathrm{~K}$ [10], while tetragonal $\mathrm{FeS}$ has been reported to be a superconductor with $T_{c} \sim 5 \mathrm{~K}$ [11]. Moreover, superconductivity in FeSe can be boosted to $\sim 40 \mathrm{~K}$ and even to about $100 \mathrm{~K}$ by means of K-intercalation [12] and in the case of a monolayer of FeSe on $\mathrm{SrTiO}_{3}$ 13. Interestingly, while $\mathrm{FeSe}$ is not magnetically long-range ordered, magnetism appears both upon compression (most probably consistent with a stripe-type $(\pi, \pi)$ vector [14]) and upon expansion. For example, the end-member FeTe has a long-range $(\pi, 0)$ antiferromag- 
netic order [15]. These findings clearly demonstrate that isoelectronic tuning of the lattice of FeSe, e.g., by doping, can be an effective means to control $T_{c}$ in FeCh.

On the experimental and theoretical sides, much effort has been devoted to understand the electronic properties of FeCh employing, e.g., angle-resolved photoemission (ARPES) measurements [1] and theoretical band structure calculations [16]. In particular, it has been shown that FeSe has the same Fermi surface (FS) topology as the pnictides, characterized by an in-plane stripetype nesting $(\pi, \pi)$, consistent with $s^{ \pm}$pairing symmetry [17]. A significant narrowing of the Fe $3 d$ bandwidth by a factor of $\sim 2$ and a large orbital-selective enhancement of the quasiparticle mass $m^{*} / m$ in the range of $\sim 3-20$ was reported [18, 19], implying a crucial importance of (orbital-selective) electronic correlations [20 24]. In addition, recent photoemission data show a formation of the lower Hubbard-band satellite at about $-2 \mathrm{eV}$ in bulk FeSe which is absent in density functional theory (DFT) [22, 24]. Furthermore, computations of the lattice parameters of iron-based superconductors within DFT highlight peculiar deviations with experiments, not captured by standard band structure methods [23, 25]. All this suggests a complex interplay between electronic correlations and the lattice, implying the crucial importance of the effect of electronic correlations in FeCh.

State-of-the-art methods for the calculation of the electronic structure of correlated materials using band structure methods combined with dynamical mean-field theory $(\mathrm{DFT}+\mathrm{DMFT})$ [26, 27] have shown to provide a good quantitative description of the electronic structure of FeCh [23, 24, 28, 29]. In fact, recent DFT+DMFT calculations show a significant orbital-dependent mass enhancement in the range of $2-5$ and demonstrate the existence of a lower Hubbard band at about -1.5 to $-2.0 \mathrm{eV}$ below the Fermi level of FeSe [22 24, 28]. Electronic correlations in FeSe show a strong sensitivity to variation of the crystal structure parameters. For example, both $T_{c}$ and the correlation strength are found to be affected by an isostructural change of the lattice volume [30]. Indeed, upon expansion of the lattice caused by substitution of Se for Te, $T_{c}$ increases up to $\sim 14 \mathrm{~K}$. It also leads to a pronounced damping of quasiparticle coherence, i.e., to an enhancement of the strength of electronic correlations [30]. By contrast, upon compression of the lattice, e.g., by doping of FeSe with S, the electronic correlations becomes weaker, as can be expected for a correlated metal [31]. This is associated with a substantial increase of the band width, i.e., tetragonal $\mathrm{FeS}$ is less correlated than FeSe. We also note that $T_{c}$ is about $5 \mathrm{~K}$ in bulk FeS, which is close to $\sim 8 \mathrm{~K}$ in FeSe [11]. Interestingly, it has been recently proposed that in FeS the electron-phonon interaction is significant 32.

Moreover, the above-mentioned changes of the lattice volume have a strong effect on the FS. In fact, in $\mathrm{Fe}\left(\mathrm{Se}_{1-x} \mathrm{Te}_{x}\right)$, i.e., upon expansion of the lattice, experiments observe a suppression of the spectral weight associated with the FS pockets near the $M$ point of the
Brillouin zone [19]. This behavior is accompanied by the appearance of spectral weight near the $\mathrm{X}$ point, indicating the existence of a topological change of the FS (Lifshitz transition). We note that this effect as well as a $(\pi, \pi)$ to $(\pi, 0)$ reconstruction of magnetic correlations upon going from FeSe to FeTe, has been well established by recent DFT + DMFT calculations [23]. Furthermore, compression of the lattice due to chemical (positive) pressure cause a damping of the spectral weight associated with the inner-most FS hole pocket at the $\Gamma$ point [33]. This suggests the possibility of superconductivity in FeCh without coherent quasiparticles in a hole band forming the inner cylinder of the FS. Overall, these results reveal a subtle interplay between the electronic, magnetic and structural properties of FeSe and demonstrate the crucial importance of electronic correlations.

In our previous reports [23] based on the DFT+DMFT approach, we studied the electronic structure and phase stability of FeSe under negative pressure which can be achieved experimentally by doping FeSe with Te. In agreement with experiment FeSe was shown to exhibit a remarkable change of the electronic structure and the magnetic and lattice properties upon expansion of the lattice. Namely, a structural transition from a collapsedtetragonal to tetragonal phase was found to occur, which is accompanied by a reconstruction of the electronic structure (Lifshitz transition) and by a $(\pi, \pi)$ to $(\pi, 0)$ change of magnetic correlations. Furthermore there is a crossover from Fermi-liquid to non-Fermi-liquid behavior [23]. At the same time the synergy of electronic structure, magnetism, and the lattice of FeSe under external pressure has remained essentially unexplored. For that reason we will address this question in our present investigation to provide a microscopic explanation of the properties of FeSe under pressure.

In this paper, we focus on the interplay between the electronic structure and the magnetic and structural properties of the tetragonal $(P 4 / \mathrm{nmm})$ FeSe under external (positive) pressure. In particular, we study the effect of pressure on the unit cell shape, band structure, magnetic properties, and the Fermi surface of FeSe, employing a fully charge self-consistent implementation of the DFT+DMFT method [34]. We perform a structural optimization and compute the evolution of the lattice parameters, i.e., of the volume, the $c / a$ ratio, the internal $z$ position of Se (which is related to the height of Se above the Fe plane), and the electronic structure of the tetragonal unit cell (space group $P 4 / \mathrm{nmm}$ ) of paramagnetic FeSe. Our results demonstrate that electronic correlations need to be taken into account in the structural optimization of tetragonal FeSe at ambient and positive pressure. We show that the lattice parameters of FeSe obtained within DFT+DMFT, i.e., when taking into account the effect of electronic correlations, are in good agreement with experimental data. Most importantly, our results reveal a topological change of the Fermi surface (Lifshitz transition) which is accompanied by a twoto three-dimensional crossover. We obtain a small reduc- 
tion of the quasiparticle mass renormalization $m^{*} / m$ by about $5 \%$ for the $e$ and less than $1 \%$ for the $t_{2}$ states, as compared to ambient pressure. While the behavior of the momentum-resolved static susceptibility $\chi(\mathbf{q})$ shows no topological changes of magnetic correlations under pressure, it demonstrates a reduction of the degree of the in-plane $(\pi, \pi)$ stripe-type nesting. Our results for the electronic structure and lattice parameters of FeSe under pressure are in line with recent experiments on its isoelectronic and isostructural counterpart $\mathrm{FeSe}_{1-x} \mathrm{~S}_{x}$. Overall, our results show the sensitivity of the electronic structure and magnetic properties of $\mathrm{FeCh}$ with respect to a small variation of the lattice. This is associated with a peculiarity of its correlated band structure, namely the proximity of a van Hove singularity associated with the $x y$ and $x z / y z$ Fe $3 d$ orbitals to the Fermi level [23, 35].

\section{METHOD}

To study the electronic structure, spectral properties, and phase stability of bulk FeSe under pressure we make use of the DFT+DMFT computational approach [27]. We employ a fully charge self-consistent DFT+DMFT scheme [34] and perform direct structural optimization of the tetragonal (space group $P 4 / \mathrm{nmm}$ ) unit cell of FeSe, calculating the total energy as a function of volume, lattice parameter $c / a$, and the internal $z_{\text {Se }}$ position. For simplicity $z_{\mathrm{Se}}$ was fixed to its experimental value $z_{\mathrm{Se}}=0.266$ [6] in some of these calculations. In order to determine the pressure, we fit our total-energy results to the third-order Birch-Murnaghan equation of state [36]. The calculations are performed for the tetragonal $(P 4 / \mathrm{nmm})$ crystal structure of paramagnetic FeSe at an electronic temperature $T=290 \mathrm{~K}$. We note that according to experimental data, bulk FeSe undergoes a tetragonal-to-orthorhombic (nematic) phase transition at sufficiently lower temperature $\sim 90 \mathrm{~K}$ (at ambient pressure) [1, 6, 7].

The DFT+DMFT approach is implemented within plane-wave pseudopotentials with the generalized gradient approximation in DFT [37]. For the partially filled $\mathrm{Fe} 3 d$ and Se $4 p$ orbitals we construct a basis set of Wannier functions using the projection procedure on a local atomic-centered symmetry-constrained basis set within a window spanning both the Fe $3 d$ and Se $4 p$ bands [38]. To solve the realistic many-body problem, we employ the continuous-time hybridization-expansion (segment) quantum Monte Carlo algorithm [39]. In accordance with previous studies of the pnictides and chalcogenides, we use the average Coulomb interaction $U=3.5 \mathrm{eV}$ and Hunds exchange $J=0.85 \mathrm{eV}$ parameters for the Fe $3 d$ orbitals [23, 40]. The $U$ and $J$ values are assumed to remain constant upon variation of the lattice volume. The Coulomb interaction is treated in the density-density approximation. Spin-orbit coupling is neglected in these calculations. Furthermore, we employ the fully localized double-counting correction, evaluated from the self- consistently determined local occupations, to account for the electronic interactions already described by DFT. To compute spectral properties and renormalizations of the effective electron mass of the $\mathrm{Fe} 3 d$ orbitals, we calculate the real-axis self-energy $\Sigma(\omega)$ using Padé analytical continuation procedure [41]. The evolution of magnetic correlations under pressure is analyzed by calculating the momentum-resolved static susceptibility in the particlehole bubble approximation:

$$
\chi(\mathbf{q})=-k_{\mathrm{B}} T \sum_{\mathbf{k}, i \omega_{n}} \operatorname{Tr} \hat{G}\left(\mathbf{k}, i \omega_{n}\right) \hat{G}\left(\mathbf{k}+\mathbf{q}, i \omega_{n}\right),
$$

where $T$ is the temperature, $\omega_{n}$ is the fermionic Matsubara frequency and $\hat{G}\left(\mathbf{k}, i \omega_{n}\right)$ is the interacting lattice Green's function computed within DFT+DMFT.

\section{RESULTS}

\section{A. Crystal structure optimization}

We start by performing a structural optimization of tetragonal $(P 4 / \mathrm{nmm})$ FeSe at ambient pressure. To this end, we compute the total energy as a function of volume, lattice parameter $c / a$, and the internal position of Se $z_{\text {Se }}$ (the space-group symmetry remains unchanged, $P 4 / n m m)$ using the charge self-consistent DFT+DMFT method. The equilibrium structural parameters are determined by calculating the minimum of the total energy functional. Our results are summarized in Fig. 1. We note that the equilibrium lattice parameters of FeSe computed within the nonmagnetic GGA (nm-GGA) using the plane-wave pseudopotential approach, i.e., $V=$ 563 a.u. ${ }^{3}, c / a=1.671$ and $z_{\mathrm{Se}}=0.227$, are substantially (by $\sim 6-15 \%$ ) off the experimental values; this is out of the range of the plot of the $c / a$ ratio vs. volume shown in Fig. 1 [6, 42]. Fixing the $z$ position of Se to its experimental value $z_{\mathrm{Se}}=0.266$, the equilibrium lattice volume and the $c / a$ ratio values calculated within nm-GGA are 485 a.u. ${ }^{3}$ and 1.471, respectively, which is about $8 \%$ lower (volume) and $\sim 1 \%$ larger $(c / a)$ than those found in experiment (see Fig. 10).

By contrast, our results for the equilibrium lattice parameters obtained by DFT+DMFT agree well with experiment. In particular, we find the equilibrium volume as 511 a.u. ${ }^{3}$ and the $c / a$ ratio as 1.494 , which agree within $3 \%$ with the experimental values. The calculated Se $z$ fractional position $z_{\mathrm{Se}}=0.261$ is in excellent agreement with experiment [ $[6]$. Furthermore, while in nm-GGA the results for the lattice parameters obtained with and without optimization of $z_{\mathrm{Se}}$ strongly differ, this is not the case within DFT+DMFT. Our result for the (instantaneous) local magnetic moment $\sqrt{\left\langle\hat{m}_{z}^{2}\right\rangle} \sim 1.86 \mu_{\mathrm{B}}$ is close to that found in the previous charge self-consistent DFT + DMFT calculations 23] with $c / a$ fixed to its experimental value. The calculated bulk modulus is $K_{0} \sim 85 \mathrm{GPa}$, which is comparable with that for iron pnictides. We note that 


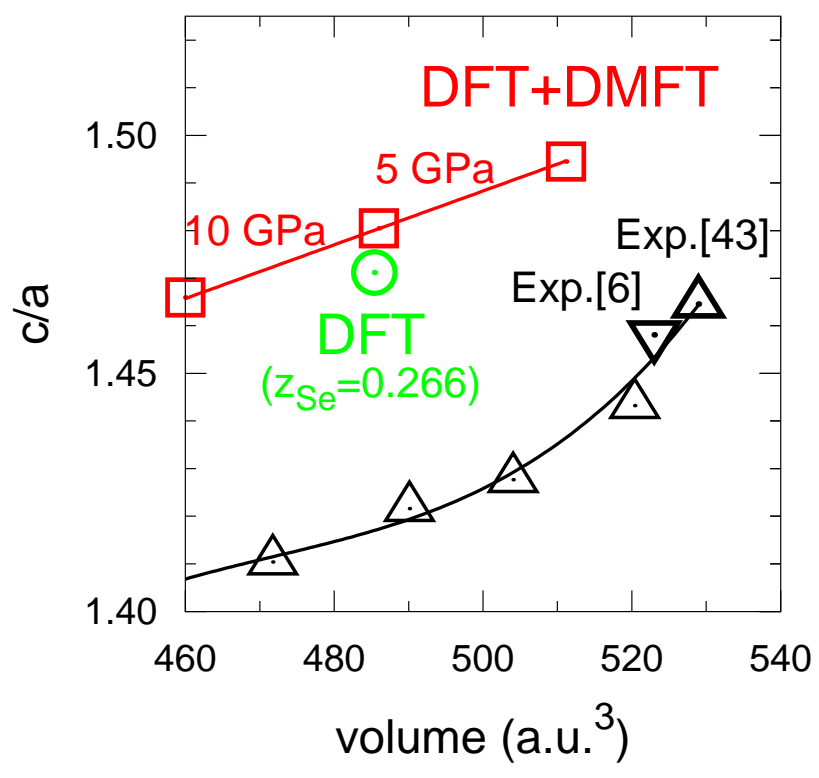

FIG. 1: (Color online) Evolution of the lattice volume and $c / a$ ratio of paramagnetic tetragonal FeSe under pressure obtained by DFT+DMFT at $T=290 \mathrm{~K}$ (squares) as compared to the experimental results (triangles [42], inverted triangle [6]). Bold triangles mark the cell parameters at ambient pressure. The nm-GGA result with $z_{\text {Se }}$ fixed to its experimental value is shown by a circle. Note that a fully relaxed result obtained within nm-GGA would be out of the range of the plot (see text).

this is much lower than the result obtained without electronic correlations (116 GPa).

Moreover, we observe that the $c / a$ ratio obtained within DFT+DMFT is slightly overestimated by about $3 \%$. This is presumably related to the van der Waals (attractive) interaction between the electrically neutral FeSe layers, which is not taken into account in our calculations. It has been shown previously that in the binary chalcogenides the local (repulsive) Coulomb interaction is important for calculation of the in-plane lattice constant and the volume of the unit cell 23]. On the other hand, the van der Waals attraction was found to correct the overestimation of the out-of-plane lattice parameter in DFT [43].

Next, we calculate the evolution of the lattice parameters of FeSe under pressure (see Fig. 11). For simplicity, in these calculations the internal coordinate $z_{\text {Se }}$ was fixed at its experimental value which is very close to that obtained within DFT+DMFT at ambient pressure. Furthermore, for subsequent verification we perform an optimization of $z_{\text {Se }}$ at pressure $\sim 10 \mathrm{GPa}$, which results in a weak $\sim 2 \%$ increase of $z_{\mathrm{Se}}$, in agreement with experiment [42]. In Fig. 1 we display our results for the evolution of the $c / a$ ratio and the lattice volume of FeSe upon compression. Our calculations exhibit a monotonous decrease of the $c / a$ ratio and the lattice volume of FeSe, which can be ap-
TABLE I: Calculated structural parameters of tetragonal FeSe at ambient pressure and those at about $\sim 10 \mathrm{GPa}$ as obtained by nm-GGA and DFT+DMFT in comparison with experiment [6, 42]. Here $h_{\mathrm{Se}}$ is the height of Se above the basal Fe plane, $d_{\mathrm{Fe}-\mathrm{Se}}$ and $d_{\mathrm{Fe}-\mathrm{Fe}}$ are the distances between the corresponding ions. a.p. - results obtained at ambient pressure.

\begin{tabular}{|c|c|c|c|c|c|c|}
\hline $\begin{array}{c}p \\
(\mathrm{GPa})\end{array}$ & $\begin{array}{c}V \\
\left(\text { a.u. }^{3} \text { ) }\right. \\
\end{array}$ & $c / a$ & $z_{\mathrm{Se}}$ & $\begin{array}{c}d_{\mathrm{Fe}-\mathrm{Se}} \\
\text { (a.u.) }\end{array}$ & $\begin{array}{c}d_{\mathrm{Fe}-\mathrm{Fe}} \\
\text { (a.u.) }\end{array}$ & $\begin{array}{l}h_{\mathrm{Se}} \\
\text { (a.u.) }\end{array}$ \\
\hline & \multicolumn{6}{|c|}{$\overline{\mathrm{DFT}+\mathrm{DMFT}}$} \\
\hline a.p. & 511 & 1.494 & 0.261 & 4.438 & 4.950 & 2.729 \\
\hline 10 & 460 & 1.464 & 0.280 & 4.395 & 4.807 & 2.787 \\
\hline & \multicolumn{6}{|c|}{ nm-GGA } \\
\hline a.p. & 563 & 1.671 & 0.227 & 4.366 & 4.920 & 2.638 \\
\hline 10 & 433 & 1.402 & 0.282 & 4.307 & 4.781 & 2.670 \\
\hline & \multicolumn{6}{|c|}{ Exp. (room temperature) } \\
\hline a.p. [6] & 523 & 1.458 & 0.266 & 4.498 & 5.024 & 2.759 \\
\hline a.p. [42] & 529 & 1.464 & 0.288 & 4.657 & 5.036 & 3.001 \\
\hline $8.5[42]$ & 456 & 1.406 & 0.319 & 4.614 & 4.858 & 3.080 \\
\hline 11 [42] & 447 & 1.397 & 0.306 & 4.487 & 4.824 & 2.915 \\
\hline
\end{tabular}

proximated (up to accuracy of the present DFT+DMFT calculations) by a straight line. The slope of this line, i.e., the relative dependence of the $c / a$ on the lattice volume, is in good agreement with experimental data for the unit cell parameters at $2-11 \mathrm{GPa}$ [42]. However, the experimental data show a substantial change (kink) in the slope of the $c / a$ ratio vs. lattice volume at about $2 \mathrm{GPa}\left(V \sim 520\right.$ a.u. $\left.{ }^{3}\right)$, which is not reproduced in our DFT+DMFT calculations. We attribute this discrepancy to the van der Waals attraction between the FeSe layers, which is not included in the present calculations.

Our results for the lattice parameters of FeSe under pressure are in overall good agreement with recent experimental data [42]. Moreover, our calculations demonstrate the crucial importance of electronic correlations in determining the structural properties of FeSe under pressure. For a pressure of $\sim 10 \mathrm{GPa}$, we obtain a lattice volume $V \sim 460$ a.u. ${ }^{3}$ and a ratio $c / a=1.464$, which is $<1 \%$ larger than the lattice volume and about $4 \%$ larger than $c / a$ observed in experiment (see Table I), respectively. Our result for the local magnetic moment $1.82 \mu_{B}$ is close to the result obtained at ambient pressure.

\section{B. Spectral properties and Fermi surface under pressure}

We now compute the evolution of the spectral properties of FeSe under pressure. Our results for the Fe $3 d$ spectral functions obtained by DFT+DMFT at ambient pressure and upon compression to $\sim 10 \mathrm{GPa}$ are shown in Fig. 2. The former results are in good quantitative agreement with our previous DFT+DMFT study of FeSe [23]. In particular, all five $\mathrm{Fe} 3 d$ orbitals form a band with a predominant contribution at the Fermi level originating from the $t_{2}$ states. Moreover, in accor- 


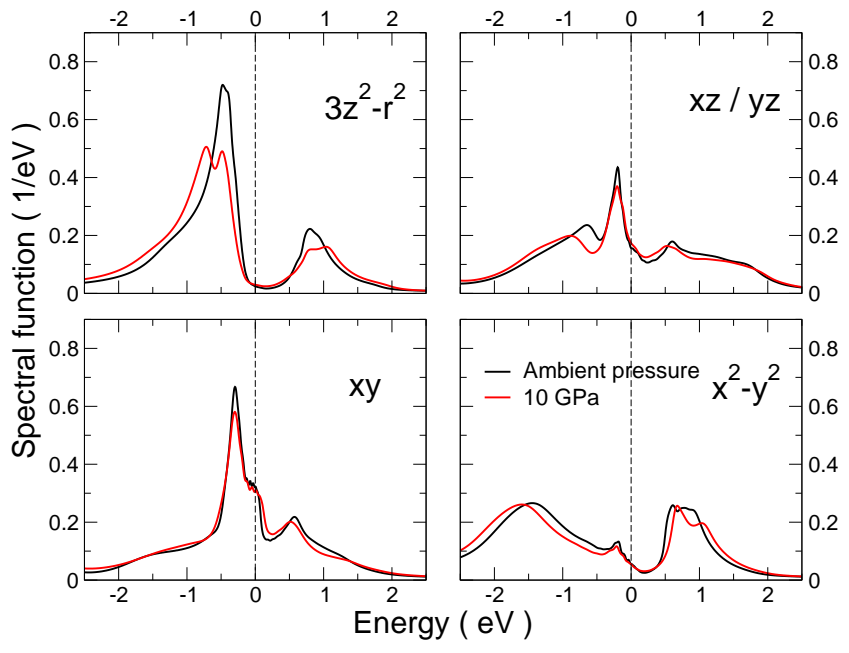

FIG. 2: (Color online) Orbitally-resolved Fe $3 d$ spectral functions of FeSe obtained within DFT+DMFT at $T=290 \mathrm{~K}$ for ambient pressure and for about $10 \mathrm{GPa}$. The Fermi energy $\left(E_{\mathrm{F}}=0 \mathrm{eV}\right)$ is shown by a vertical line.

dance with previous investigations, we observe a reduction of the Fe $3 d$ bandwidth (in comparison to the DFT results) near the Fermi energy caused by electronic correlations. The lower Hubbard band associated with the Fe $d_{x y}$ and $d_{x z / y z}$ orbitals is located at about $-1.5 \mathrm{eV}[22$ 24]. The Fe $d_{x y}$ and $d_{x z / y z}$ spectral functions exhibit a well-defined quasiparticle peak located below the Fermi level at about $0.2 \mathrm{eV}$, originating from the van Hove singularity of the $d_{x y}$ and $d_{x z / y z}$ bands at the $\mathrm{M}$ point. Upon compression, we find no substantial spectral weight transfer, in remarkable contrast to the DFT + DMFT results obtained upon expansion of the lattice. Our results exhibit a weak increase of the bandwidth and a subtle splitting of the peaks associated with the $e$ states located at $\sim 1 \mathrm{eV}$ above and below the Fermi level. However, the overall shape of the spectral functions shows only a weak pressure dependence. Under pressure, our results reveal no substantial reduction of the $\mathrm{Fe} 3 d$ spectral weight at the Fermi level, which is less than $1 \%$. These results agree well with an analysis of the band mass enhancement $m^{*} / m=1-\partial \operatorname{Re} \Sigma(\omega) /\left.\partial \omega\right|_{\omega=0}$, which provides a quantitative measure of the correlation strength. We calculate the derivative $\partial \operatorname{Re} \Sigma(\omega) /\left.\partial \omega\right|_{\omega=0}=\partial \operatorname{Im} \Sigma(i \omega) /\left.\partial i \omega\right|_{i \omega=0}$ employing Padé extrapolation of the self-energy $\Sigma(\omega)$ to $\omega=0 \mathrm{eV}$. In particular, under pressure of $\sim 10 \mathrm{GPa}$ we obtain a $\sim 5 \%$ reduction of $m^{*} / m$ for the $e$ states and less than $1 \%$ for the $t_{2}$ states, as compared to the ambient pressure.

To proceed further, we calculate the $\mathbf{k}$-resolved spectral functions of FeSe. In Fig. 3 we present our results for the Fermi surface (FS) of FeSe calculated for $k_{z}=0$ and $k_{x}=k_{y}$ by integration of the spectral weight $A(\mathbf{k}, \omega) \equiv-\frac{1}{\pi} \operatorname{Im}[G(\mathbf{k}, \omega)]$ over a $\pm 5 \mathrm{meV}$ energy window around the Fermi level. In agreement with previous studies, at ambient pressure we find two intersecting elliptical electron Fermi surfaces centered at the Brillouin zone M

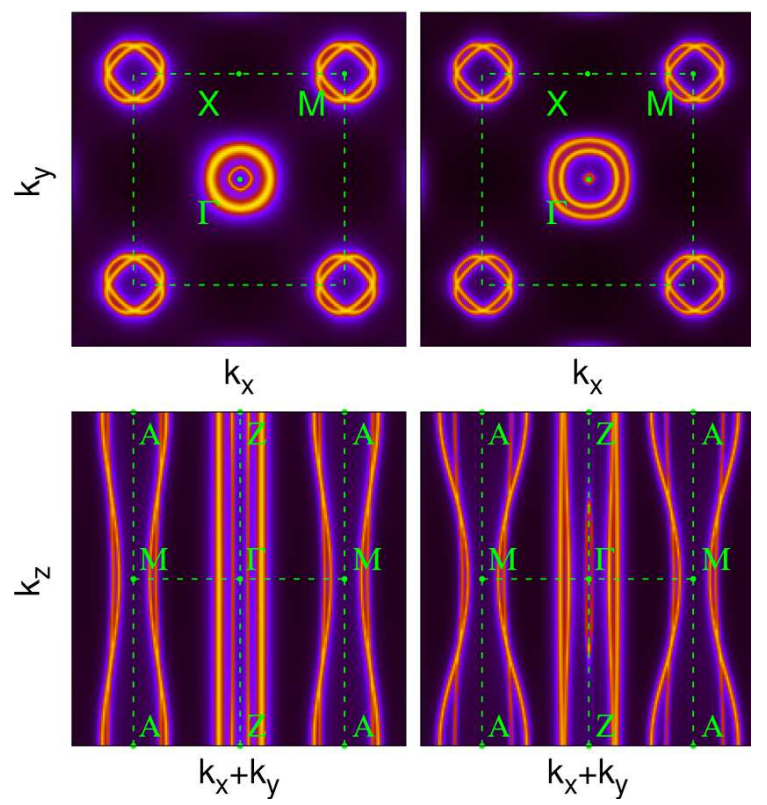

FIG. 3: (Color online) Fermi surface sections in the $k_{z}=0$ (top) and $k_{x}=k_{y}$ (bottom) planes of the tetragonal Brillouin zone of FeSe at ambient pressure (left column) and at $p=10 \mathrm{GPa}$ (right column) as computed by DFT+DMFT at $T=290 \mathrm{~K}$. Under pressure the inner hole-like Fermi surface centered at the $\Gamma$ point collapses halfway between the $\Gamma$ and $\mathrm{Z}$ points, resulting in a closed elliptic-like three-dimensional FS (bottom-right panel).

point, associated with the $d_{x y}$ and $d_{x z / y z}$ orbitals. In addition, there are three concentric hole-like pockets at the $\Gamma$ point (the two outer hole pockets are degenerate). The FS is seen to be remarkably expanded as compared to the prediction of the low-temperature ARPES measurements [1, 18, 19]. It was indeed proposed that this is related to a "blue-red shift" problem [35], i.e., unappropriate shifting upward of the electron bands at the $\mathrm{M}$ and downward of the hole-like bands at the $\Gamma$ point, presumably due to a momentum dependence of the real part of self-energy $\Sigma(\mathbf{k}, \omega)$ (the latter by construction is local, k-independent $\Sigma(\omega)$, in our single-site DFT + DMFT calculations). We note however that our DFT + DMFT calculations are carried out at $T=290 \mathrm{~K}$, whereas a direct comparison of our result for the FS with recent room-temperature ARPES measurements [35] shows good quantitative agreement.

We find that bulk FeSe is a correlated metal with coherent quasiparticle behavior at the Fermi level (welldefined FS), which exhibits incoherent spectral weight at higher binding energies, in agreement with recent experiments [35]. We note that at ambient pressure, a structural optimization including relaxation of the $z_{\mathrm{Se}}$ coordinate neither affects the topology of the FS nor the in-plane nesting vector $(\pi, \pi)$, connecting the electronlike and hole-like FS pockets. The latter is indicative of stripe-type spin excitations, consistent with a $s^{ \pm}$pairing symmetry [17]. We also note that the size of the FS pock- 


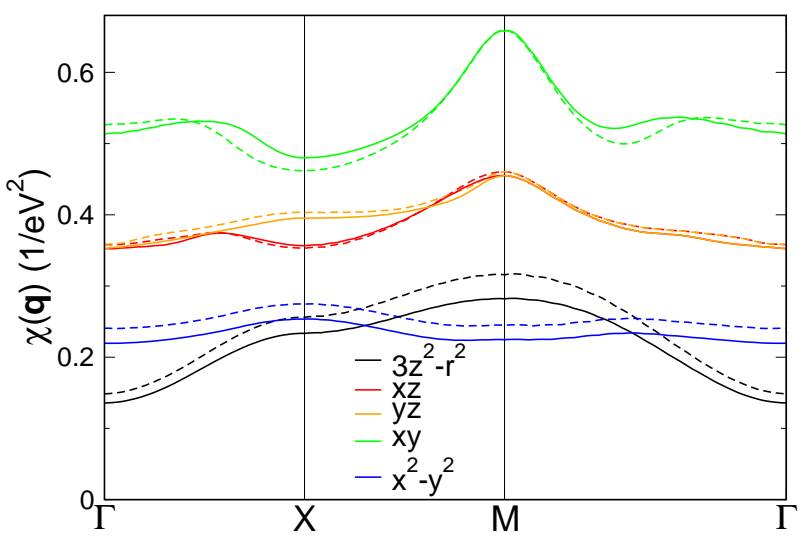

FIG. 4: (Color online) Orbital contributions to the static spin susceptibility $\chi(\mathbf{q})$ of paramagnetic FeSe at ambient pressure (broken line) and $p=10 \mathrm{GPa}$ (solid line) calculated along the $\Gamma-\mathrm{X}-\mathrm{M}-\Gamma$ path using DFT+DMFT.

ets depends only weakly on the structural optimization of the lattice.

Upon compression of the FeSe lattice, we observe a remarkable reconstruction of the electronic structure and of the FS, i.e., a Lifshitz transition (see Fig. 3, right panel). In particular, the inner hole-like FS centered at the $\Gamma$ point collapses halfway between the $\Gamma$ and $\mathrm{Z}$ points, resulting in a closed elliptic-like three-dimensional FS, elongated in the $k_{z}$ direction, around $\Gamma$. At the same time, the topology of the electron-like FS at the M point shows no qualitative change, while its cross-section in the $\left(k_{x}, k_{y}\right)$ plane now exhibits a substantial variation for different $k_{z}$. In fact, the elongation of the FS ellipses in the $\left(k_{x}, k_{y}\right)$ cross-section is small for $k_{z}=0$ and large for $k_{z}=\pi$. Overall, our results clearly show that bulk FeSe under pressure undergoes a Lifshitz transition which is associated with a two- to three-dimensional crossover of the FS.

Bulk FeSe at about $10 \mathrm{GPa}$ is seen to be a moderately correlated metal with coherent quasiparticle behavior at the Fermi level. The latter makes the electronic properties of FeSe particularly interesting since, both under positive and negative (chemical) pressure, it exhibits a Lifshitz transition. Interestingly, according to experiments $T_{c}$ is boosted up to $\sim 37 \mathrm{~K}$ in FeSe under pressure of about $9 \mathrm{GPa}$ [5] and up to $\sim 17 \mathrm{~K}$ in $\mathrm{FeSe}_{1-x} \mathrm{Te}_{x}$ upon negative chemical pressure caused by substituting Se by Te [10]. However, on a qualitative level, the Lifshitz transition upon positive pressures does not result in a coherence-incoherence crossover as it was found upon expansion of the lattice in Refs. 19, 23].

The change of the FS results in a corresponding change of magnetic correlations due to a suppression of the inplane nesting vector $Q_{m}=(\pi, \pi)$. In particular, our results exhibit a small reduction of the magnetic correlations under (positive) pressure, i.e., $\chi(\mathbf{q})$ shows a small decrease due to the $e$ states compared to the results at ambient pressure. At the same time the in-plane nesting

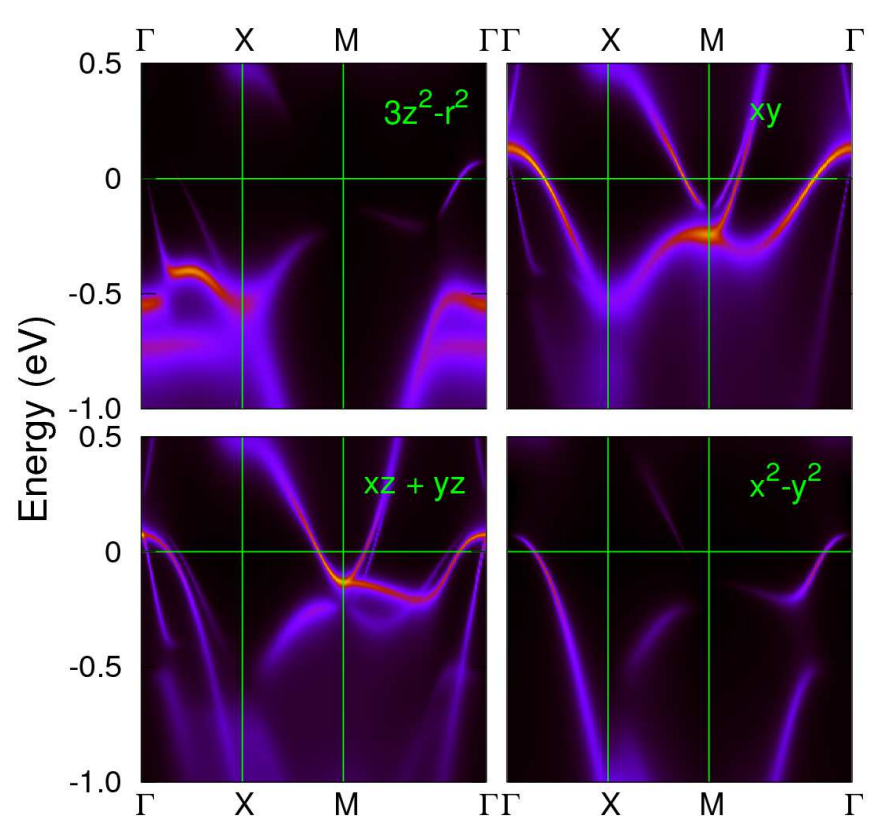

FIG. 5: (Color online) Orbitally-resolved band structure of FeSe along the $\Gamma-\mathrm{X}-\mathrm{M}-\Gamma$ path as obtained by DFT+DMFT at $T=290 \mathrm{~K}$ and $p=10 \mathrm{GPa}$.

$Q_{m}$ vector remains of the $(\pi, \pi)$-type which is consistent with stripe-type spin fluctuations (see Fig. (4). This is in stark contrast to the $(\pi, \pi)$-to- $(\pi, 0)$ reconstruction of the magnetic correlations in FeSe upon expansion of the lattice (negative chemical pressure in $\mathrm{Fe}(\mathrm{Se}, \mathrm{Te})$ ) previously found in [19, 23]. Furthermore, we note that the nm-GGA results reveal no substantial change in the FS of FeSe upon moderate compression or expansion of the lattice. This highlights once more the great importance of electronic correlations, which are found to be responsible for the remarkable reconstruction of the electronic structure of bulk FeSe, associated with a Lifshitz transition, both upon expansion and compression of the lattice volume.

To analyze our results further we calculated the electronic structure of bulk FeSe at ambient pressure and at about $10 \mathrm{GPa}$. In Fig. 5 we present our results for the orbitally-resolved spectral function evaluated in the $\Gamma-\mathrm{X}-\mathrm{M}$ plane of the Brillouin zone $\left(k_{z}=0\right)$. The total spectral functions of FeSe computed in the $\Gamma-\mathrm{X}-\mathrm{M}$ $\left(k_{z}=0\right)$ and R-Z-A $\left(k_{z}=\pi\right)$ planes are shown in Fig. 6. In agreement with previous DFT+DMFT calculations, we obtain a substantial renormalization of the effective crystal-field splitting of the Fe $3 d$ bands with respect to the DFT results caused by the strong energy and orbital dependence of the self-energy 22, 23, 28]. The latter leads to different shifts of the quasiparticle bands near the Fermi level. In particular, in agreement with the ARPES measurements, we observe a "blue-red shift" of the states near the M point (which are pushed upwards) and the hole pockets near the $\Gamma$ point (pushed downwards). Both shift towards the Fermi level (see Fig. 6) due to orbital-selective correlation effects. In addition, 


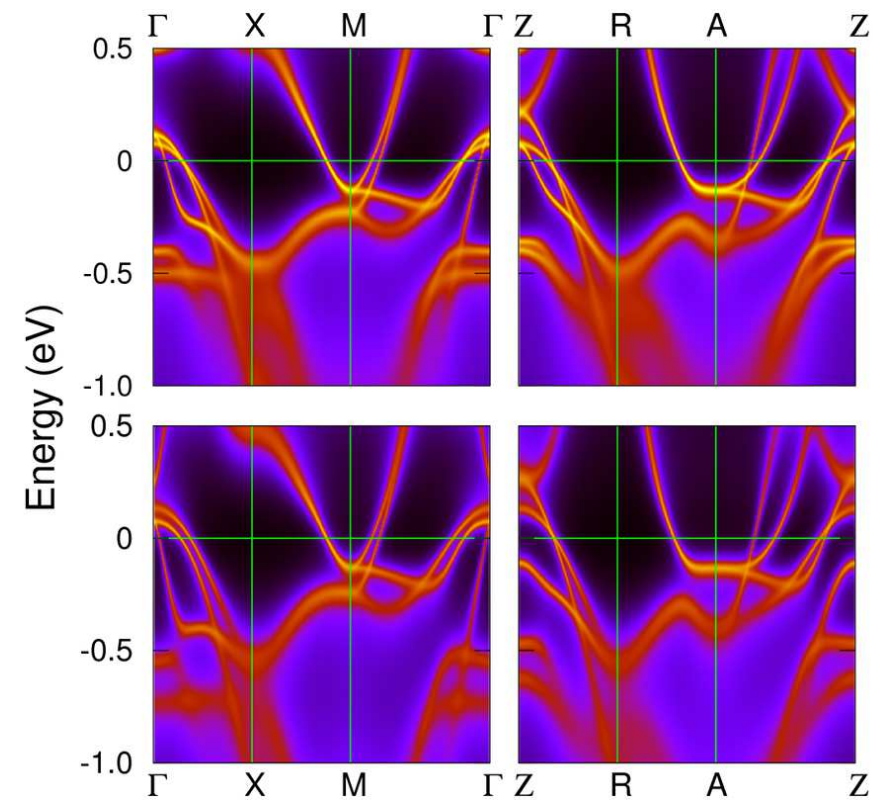

FIG. 6: (Color online) Band structure of FeSe along the $\Gamma$ $\mathrm{X}-\mathrm{M}-\Gamma$ and $\mathrm{Z}-\mathrm{R}-\mathrm{A}-\mathrm{Z}$ directions at ambient pressure (top row) and $p=10 \mathrm{GPa}$ (bottom row) as computed by DFT+DMFT at $T=290 \mathrm{~K}$.

our results clearly distinguish the van Hove singularity at the M point, associated with the Fe $d_{x y}$ and $d_{x z / y z}$ orbitals (see Fig. 5 ).

We note that at ambient pressure the spectral function of FeSe evaluated in the $\Gamma-\mathrm{X}-\mathrm{M}\left(k_{z}=0\right)$ and $\mathrm{R}$ Z-A $\left(k_{z}=\pi\right)$ planes (see Fig. 6] top row) exhibits the same structure. It consists of three hole-like bands near the center (at the $\Gamma$ and $\mathrm{Z}$ points) and two electron-like bands near the Brillouin zone corners (at the $\mathrm{M}$ and $\mathrm{A}$ points). By contrast, under pressure of $\sim 10 \mathrm{GPa}$, we observe an orbital-dependent shift of the energy bands near the $\mathrm{Z}$ point, resulting in a topological change of the FS (Lifshitz transition), along the $\Gamma-\mathrm{Z}$ (R-Z) direction. In particular, the $x y$ band shifts towards the Fermi level in the $k_{z}=0$ plane but still remains above the Fermi level (at the $\Gamma$ point). On the other hand, for $k_{z}=\pi$ the $x y$ band is pushed below the Fermi level (near the Z point), resulting in the formation of a closed inner hole pocket in the $\Gamma-\mathrm{Z}$ direction. Therefore we conclude that a microscopic mechanism of the Lifshitz transition which results in a 2D-3D dimensional crossover in $P 4 / \mathrm{nmm}$ FeSe under pressure is associated with a pressure-induced shift of the $d_{x y}$-derived band. We note that the $x y$ band exhibits the largest renormalization of the quasiparticle mass $m^{*} / m$ 22 24, 28]. Overall, our results show that renormalizations of the band structure of FeSe under pressure are weaker than those at ambient pressure as it is expected for a typical correlated metal.

The reconstruction of the electronic structure and the evolution of the FS of FeSe under pressure agree reasonably well with available experiments. Moreover, our results are in line with the experimentally observed ex- pansion of the FS of the $\mathrm{FeSe}_{1-x} \mathrm{~S}_{x}$ series upon addition of $\mathrm{S}$ [31, 33]. In fact, tetragonal $\mathrm{FeS}$ is an isoelectronic counterpart of $\mathrm{FeSe}$ with a smaller lattice volume, due to a smaller ionic radius of $\mathrm{S}$ with respect to Se. Therefore the properties of $\mathrm{FeSe}_{1-x} \mathrm{~S}_{x}$ can be regarded as those corresponding to FeSe under positive pressure. With this in mind, our results imply that FeSe under pressure and, hence, $\mathrm{FeSe}_{1-x} \mathrm{~S}_{x}$ are less correlated than FeSe, in agreement with recent studies 31]. An analysis of the FSs and magnetic correlations of FeSe under pressure in terms of $\chi(\mathbf{q})$ suggests that $\mathrm{FeSe}_{1-x} \mathrm{~S}_{x}$ exhibits stripe-type spin excitations with in-plane $(\pi, \pi)$ nesting. The latter are weakened by the damping of spin fluctuations upon compression of the lattice [33].

Under pressure, our results reveal the three hole-like pockets of the FS in the vicinity of the $\Gamma$ point, with the inner hole-like FS pocket being of a 3D character. However, upon further compression it is seen that the three hole-like FSs collapse to the two FSs, in agreement with recent studies of $\mathrm{FeS}$ 44. Our results suggest that the pressure-induced increase of the critical temperature in FeSe, which is boosted to $37 \mathrm{~K}$ by applying a pressure of about $9 \mathrm{GPa}[5]$, is not of spin-fluctuation origin. Indeed, it indicates that the anomalous behavior of FeSe upon variation of the lattice volume (both compression and expansion) is associated with a crossover between superconductivity mediated by spin-fluctuations (upon expansion of the lattice in $\mathrm{Fe}(\mathrm{Se}, \mathrm{Te})$ ) and by phonons (upon compression), respectively. While the low-pressure superconducting phase of FeSe most likely has an $s^{ \pm}$order parameter, one may expect a change of the symmetry, e.g., to a $d$-wave order parameter in the high pressure phase [17, 45].

\section{CONCLUSION}

By employing a fully charge self-consistent implementation of the DFT+DMFT method we performed structural optimization and studied the electronic properties of the tetragonal $(P 4 / n m m)$ phase of paramagnetic FeSe under pressure at $T=290 \mathrm{~K}$. In agreement with previous studies our results demonstrate that electronic correlations need to be included to determine the correct lattice parameters of FeSe both in the equilibrium and upon compression [23]. Our results for the lattice parameters are in overall good agreement with experimental data. At ambient pressure, the calculated $c / a$ ratio is about $3 \%$ larger than the experimental value, suggesting a possible importance of van der Waals attraction between the FeSe layers, which are absent in the present calculations. Upon compression to $10 \mathrm{GPa}$, the $c / a$ ratio and lattice volume show a linear-like decrease by $2 \%$ and $10 \%$, respectively, while the fractional coordinate of Se $z_{\mathrm{Se}}$ weakly increases by $\sim 2 \%$.

Our results for the spectral properties exhibit a rather weak dependence of the bandwidth on the lattice volume, associated with a small change of the Fe-Se hybridization 
upon a moderate compression of the lattice up to $10 \mathrm{GPa}$. Most importantly, we obtain a drastic reconstruction of the Fermi surface (FS) topology, i.e., a Lifshitz transition, indicating a two- to three-dimensional crossover in the FS. In particular, the inner hole-like pocket at the $\Gamma$ point of the FS collapses halfway between the $\Gamma$ and $\mathrm{Z}$ points, showing a three-dimensional character. We conclude that a microscopic mechanism of the Lifshitz transition is associated with a pressure-induced shift of the $d_{x y}$ orbital in the $\mathrm{Z}$ point below the Fermi level. Our results are in good agreement with recent ARPES measurements for the $\mathrm{FeSe}_{1-x} \mathrm{~S}_{x}$ solid solution, which is regarded as an isoelectronic and isostructural (with a smaller lattice volume due to chemical pressure) counterpart of FeSe. Under pressure, we observe a small reduction of the quasiparticle mass renormalization $m^{*} / m$ by about $5 \%$ for the $e$ and less than $1 \%$ for the $t_{2}$ states, as compared to results at ambient pressure. This implies that $\mathrm{FeSe}_{1-x} \mathrm{~S}_{x}$ is less correlated than $\mathrm{FeSe}$, in agreement with recent studies [31, 33, 44].

The behavior of the momentum-resolved magnetic susceptibility $\chi(\mathbf{q})$ under pressure shows no topological change of magnetic correlations under pressure.
The latter suggests that both FeSe under pressure and $\mathrm{FeSe}_{1-x} \mathrm{~S}_{x}$ exhibit stripe-type spin excitations. Moreover, an analysis of the FS and magnetic correlations exhibits a small reduction of the degree of the in-plane $(\pi, \pi)$ nesting, which implies that magnetic correlations are weakened due to the damping of spin fluctuations under pressure. We speculate that the anomalous behavior of FeSe upon variation of the lattice volume, both under expansion and compression, is associated with a crossover between superconductivity mediated by spinfluctuations (upon expansion of the lattice in $\mathrm{Fe}(\mathrm{Se}, \mathrm{Te})$ ) and by phonons (upon compression), respectively.

\section{ACKNOWLEDGMENTS}

The work was supported by the Russian Scientific Foundation (Project No. 14-22-00004). S.L.S., V.I.A., and I.L. are grateful to the Center for Electronic Correlations and Magnetism, University of Augsburg, for hospitality.
[1] X. Liu, L. Zhao, S. He, J. He, D. Liu, D. Mou, B. Shen, Y. Hu, J. Huang, and X. J. Zhou, J. Phys.: Condens. Matter 27, 183201 (2015); Y. V. Pustovit and A. A. Kordyuk, Low Temp. Phys. 42, 995 (2016); A. I. Coldea and M. D. Watson, Annu. Rev. Condens. Matter Phys. 9, 125 (2018). A. E. Böhmer and A. Kreisel, J. Phys.: Condens. Matter 30, 023001 (2018).

[2] J. Paglione and R. L. Greene, Nat. Phys. 6, 645 (2010); D. N. Basov and A. V. Chubukov, Nat. Phys. 7, 272 (2011); G. R. Stewart, Rev. Mod. Phys. 83, 1589 (2011); P. Dai, J. Hu, and E. Dagotto, Nat. Phys. 8, 709 (2012); Q. Si, R. Yu, and E. Abrahams, Nat. Rev. Mater. 1, 16017 (2016);

[3] Y. J. Kamihara, T. Watanabe, M. Hirano, and H. Hosono, J. Am. Chem. Soc. 130, 3296 (2008); Z. A. Ren, W. Lu, J. Yang, W. Yi, X. L. Shen, Z. C. Li, G. C. Che, X. L. Dong, L. L. Sun, F. Zhou, Z. X. Zhao, Chin.Phys. Lett. 25, 2215 (2008); X. H. Chen, T. Wu, G. Wu, R. H. Liu, H. Chen, and D. F. Fang, Nature (London) 453, 761 (2008).

[4] F. C. Hsu, J. Y. Luo, K. W. Yeh, T. K. Chen, T. W. Huang, P. M. Wu, Y. C. Lee, Y. L. Huang, Y. Yi. Chu, D. C. Yan and M. K. Wu, Proc. Natl. Acad. Sci. U.S.A. 105, 14262 (2008).

[5] S. Medvedev, T. M. McQueen, I. A. Troyan, T. Palasyuk, M. I. Eremets, R. J. Cava, S. Naghavi, F. Casper, V. Ksenofontov, G. Wortmann, and C. Felser, Nat. Mater. 8, 630 (2009); Q. Wang, Y. Shen, B. Pan, X. Zhang, K. Ikeuchi, K. Iida, A. D. Christianson, H. C. Walker, D. T. Adroja, M. Abdel-Hafiez, X. Chen, D. A. Chareev, A. N. Vasiliev, and J. Zhao, Nat. Commun. 7, 12182 (2016).

[6] S. Margadonna, Y. Takabayashi, M. T. McDonald, K. Kasperkiewicz, Y. Mizuguchi, Y. Takano, A. N. Fitch, E. Suard, K. Prassides, Chem. Commun. (Cambridge) 43,
5607 (2008); M. C. Lehman, A. Llobet, K. Horigane, and D. Louca, J. Phys. Conf. Ser. 251, 012009 (2010).

[7] T. M. McQueen, A. J. Williams, P. W. Stephens, J. Tao, Y. Zhu, V. Ksenofontov, F. Casper, C. Felser and R. J. Cava, Phys. Rev. Lett. 103, 057002 (2009).

[8] M. Bendele, A. Amato, K. Conder, M. Elender, H. Keller, H.-H. Klauss, H. Luetkens, E. Pomjakushina, A. Raselli and R. Khasanov, Phys. Rev. Lett. 104, 087003 (2010); M. Bendele M, A. Ichsanow, Y. Pashkevich, L. Keller, T. Strässle, A. Gusev, E. Pomjakushina, K. Conder, R. Khasanov and H. Keller, Phys. Rev. B 85, 064517 (2012).

[9] Y. Mizuguchi, F. Tomioka, S. Tsuda, T. Yamaguchi and Y. Takano, Appl. Phys. Lett. 93, 152505 (2008); S. Margadonna, Y. Takabayashi, Y. Ohishi, Y. Mizuguchi, Y. Takano, T. Kagayama, T. Nakagawa, M. Takata and K. Prassides, Phys. Rev. B 80064506 (2009); G. Garbarino, A. Sow, P. Lejay, A. Sulpice, P. Toulemonde, M. Mezouar and M. Nunez-Regueiro, Europhys. Lett. 86 27001 (2009); S. Masaki, H. Kotegawa, Y. Hara, H. Tou, K. Murata, Y. Mizuguchi and Y. Takano, J. Phys. Soc. Japan 78063704 (2009); H. Okabe, N. Takeshita, K. Horigane, T. Muranaka and J. Akimitsu, Phys. Rev. B 81205119 (2010).

[10] M. H. Fang, H. M. Pham, B. Qian, T. J. Liu, E. K. Vehstedt, Y. Liu, L. Spinu, and Z. Q. Mao, Phys. Rev. B 78, 224503 (2008); B. C. Sales, A. S. Sefat, M. A. McGuire, R. Y. Jin, D. Mandrus, and Y. Mozharivskyj, Phys. Rev. B 79, 094521 (2009); A. Martinelli, A. Palenzona, M. Tropeano, C. Ferdeghini, M. Putti, M. R. Cimberle, T. D. Nguyen, M. Affronte, and C. Ritter, ibid. 81, 094115 (2010); V. Tsurkan, J. Deisenhofer, A. Günther, Ch. Kant, H.-A. Krug von Nidda, F. Schrettle, and A. Loidl, Eur. Phys. J. B 79, 289 (2011); U. R. Singh, S. C. White, S. Schmaus, V. Tsurkan, A. Loidl, J. Deisenhofer, 
and P. Wahl, Phys. Rev. B 88, 155124 (2013).

[11] X. F. Lai, H. Zhang, Y. Q. Wang, X. Wang, X. Zhang, J. H. Lin, and F. Q. Huang, J. Am. Chem. Soc. 137, 10148 (2015); U. Pachmayr, N. Fehn, and D. Johrendt, Chem. Commun. 52, 194 (2016).

[12] M. Burrard-Lucas, D. G. Free, S. J. Sedlmaier, J. D. Wright, S. J. Cassidy, Y. Hara, A. J. Corkett, T. Lancaster, P. J. Baker, S. J. Blundell, and S. J. Clarke, Nat. Mater. 12, 15 (2013).

[13] S. Tan, Y. Zhang, M. Xia, Z. Ye, F. Chen, X. Xie, R. Peng, D. Xu, Q. Fan, H. Xu, J. Jiang, T. Zhang, X. Lai, T. Xiang, J. Hu, B. Xie, and D. Feng, Nat. Mater. 12, 634 (2013).

[14] M. D. Lumsden, A. D. Christianson, D. Parshall, M. B. Stone, S. E. Nagler, G. J. MacDougall, H. A. Mook, K. Lokshin, T. Egami, D. L. Abernathy, E. A. Goremychkin, R. Osborn, M. A. McGuire, A. S. Sefat, R. Jin, B. C. Sales, and D. Mandrus, Phys. Rev. Lett. 102, 107005 (2009); Y. Qiu, W. Bao, Y. Zhao, C. Broholm, V. Stanev, Z. Tesanovic, Y. C. Gasparovic, S. Chang, Jin Hu, Bin Qian, Minghu Fang, and Zhiqiang Mao, Phys. Rev. Lett. 103, 067008 (2009); M. D. Lumsden, A. D. Christianson, E. A. Goremychkin, S. E. Nagler, H. A. Mook, M. B. Stone, D. L. Abernathy, T. Guidi, G. J. MacDougall, C. de la Cruz, A. S. Sefat, M. A. McGuire, B. C. Sales, and D. Mandrus, Nat. Phys. 6, 182 (2010).

[15] W. Bao, Y. Qiu, Q. Huang, M. A. Green, P. Zajdel, M. R. Fitzsimmons, M. Zhernenkov, S. Chang, M. Fang, B. Qian, E. K. Vehstedt, J. Yang, H. M. Pham, L. Spinu, and Z. Q. Mao, Phys. Rev. Lett. 102, 247001 (2009); T. J. Liu, J. Hu, B. Qian, D. Fobes, Z. Q. Mao, W. Bao, M. Reehuis, S. A. J. Kimber, K. Prokeš, S. Matas, D. N. Argyriou, A. Hiess, A. Rotaru, H. Pham, L. Spinu, Y. Qiu, V. Thampy, A. T. Savici, J. A. Rodriguez, and C. Broholm, Nat. Mater. 9, 718 (2010); O. J. Lipscombe, G. F. Chen, C. Fang, T. G. Perring, D. L. Abernathy, A. D. Christianson, T. Egami, N. Wang, J. Hu, and P. Dai, Phys. Rev. Lett. 106, 057004 (2011).

[16] A. Subedi, L. Zhang, D. J. Singh, and M. H. Du, Phys. Rev. B 78, 134514 (2008); K.-W. Lee, V. Pardo, and W. E. Pickett, Phys. Rev. B 78, 174502 (2008); L. Zhang, D. J. Singh, and M. H. Du, Phys. Rev. B 79, 012506 (2009); D. Guterding, H. O. Jeschke, and R. Valentí, Phys. Rev. B 96, 125107 (2017).

[17] I. I. Mazin, D. J. Singh, M. D. Johannes, and M. H. Du, Phys. Rev. Lett. 101, 057003 (2008); A. V. Chubukov, D. V. Efremov, and I. Eremin, Phys. Rev. B 78, 134512 (2008).

[18] A. Tamai, A. Y. Ganin, E. Rozbicki, J. Bacsa, W. Meevasana, P. D. C. King, M. Caffio, R. Schaub, S. Margadonna, K. Prassides, M. J. Rosseinsky, and F. Baumberger, Phys. Rev. Lett. 104, 097002 (2010); J. Maletz, V. B. Zabolotnyy, D. V. Evtushinsky, S. Thirupathaiah, A. U. B. Wolter, L. Harnagea, A. N. Yaresko, A. N. Vasiliev, D. A. Chareev, A. E. Böhmer, F. Hardy, T. Wolf, C. Meingast, E. D. L. Rienks, B. Büchner, and S. V. Borisenko, Phys. Rev. B 89, 220506 (2014).

[19] Z. K. Liu, R.-H. He, D. H. Lu, M. Yi, Y. L. Chen, M. Hashimoto, R. G. Moore, S.-K. Mo, E. A. Nowadnick, J. Hu, T. J. Liu, Z. Q. Mao, T. P. Devereaux, Z. Hussain, and Z.-X. Shen, Phys. Rev. Lett. 110, 037003 (2013); Z. K. Liu, M. Yi, Y. Zhang, J. Hu, R. Yu, J.-X. Zhu, R.-H. He, Y. L. Chen, M. Hashimoto, R. G. Moore, S.-K. Mo, Z. Hussain, Q. Si, Z. Q. Mao, D. H. Lu, and Z.-X. Shen,
Phys. Rev. B 92, 235138 (2015).

[20] M. Yi, Z. Liu, Y. Zhang, R. Yu, J. Zhu, J. Lee, R. Moore, F. Schmitt, W. Li, S. Riggs, J.-H. Chu, B. Lv, J. Hu, M. Hashimoto, S.-K. Mo, Z. Hussain, Z. Mao, C.-W. Chu, I. Fisher, Q. Si, Z.-X. Shen, and D. Lu, Nat. Commun. 6, 7777 (2015); L. Fanfarillo, J. Mansart, P. Toulemonde, H. Cercellier, P. L. Fèvre, F. Bertran, B. Valenzuela, L. Benfatto, and V. Brouet, Phys. Rev. B 94, 155138 (2016).

[21] D. V. Evtushinsky, M. Aichhorn, Y. Sassa, Z.-H. Liu, J. Maletz, T. Wolf, A. N. Yaresko, S. Biermann, S. V. Borisenko, and B. Büchner, arXiv:1612.02313.

[22] M. D. Watson, S. Backes, A. A. Haghighirad, M. Hoesch, T. K. Kim, A. I. Coldea, and R. Valentì, Phys. Rev. B 95, 081106(R) (2017).

[23] I. Leonov, S. L. Skornyakov, V. I. Anisimov, and D. Vollhardt, Phys. Rev. Lett. 115, 106402 (2015); S. L. Skornyakov, V. I. Anisimov, D. Vollhardt and I. Leonov, Phys. Rev. B 96, 035137 (2017).

[24] M. Aichhorn, S. Biermann, T. Miyake, A. Georges, and M. Imada, Phys. Rev. B 82, 064504 (2010).

[25] A. P. Koufos, D. A. Papaconstantopoulos, and M. J. Mehl, Phys. Rev. B 89, 035150 (2014).

[26] W. Metzner and D. Vollhardt, Phys. Rev. Lett. 62, 324 (1989); G. Kotliar and D. Vollhardt, Phys. Today 57, 53 (2004); A. Georges, G. Kotliar, W. Krauth, and M. J. Rozenberg, Rev. Mod. Phys. 68, 13 (1996).

[27] V. I. Anisimov, A. I. Poteryaev, M. A. Korotin, A. O. Anokhin, and G. Kotliar, J. Phys. Condens. Matter 9, 7359 (1997); G. Kotliar, S. Y. Savrasov, K. Haule, V. S. Oudovenko, O. Parcollet, and C. A. Marianetti, Rev. Mod. Phys. 78, 865 (2006); J. Kuneš, I. Leonov, P. Augustinský, V. Křápek, M. Kollar, and D. Vollhardt, Eur. Phys. J. Spec. Top. 226, 2641 (2017).

[28] S. Mandal, R. E. Cohen, and K. Haule, Phys. Rev. B 89, 220502(R) (2014);

[29] C. Tresca, G. Giovannetti, M. Capone, and G. Profeta, Phys. Rev. B 95, 205117 (2017).

[30] Z. K. Liu, R.-H. He, D. H. Lu, M. Yi, Y. L. Chen, M. Hashimoto, R. G. Moore, S.-K. Mo, E. A. Nowadnick, J. Hu, T. J. Liu, Z. Q. Mao, T. P. Devereaux, Z. Hussain, and Z.-X. Shen, Phys. Rev. Lett. 110, 037003 (2013); Z. K. Liu, M. Yi, Y. Zhang, J. Hu, R. Yu, J.-X. Zhu, R.-H. He, Y. L. Chen, M. Hashimoto, R. G. Moore, S.-K. Mo, Z. Hussain, Q. Si, Z. Q. Mao, D. H. Lu, and Z.-X. Shen, Phys. Rev. B 92, 235138 (2015).

[31] P. Reiss, M. D. Watson, T. K. Kim, A. A. Haghighirad, D. N. Woodruff, M. Bruma, S. J. Clarke, and A. I. Coldea, Phys. Rev. B 96, 121103(R) (2017).

[32] A. Baum, A. Milosavljević, N. Lazarević, M. M. Radonjić, B. Nikolić, M. Mitschek, Z. Inanloo Maranloo, M. Śćepanović, M. Grujić-Brojčin, N. Stojilović, M. Opel, A. Wang, C. Petrović, Z. V. Popović, and R. Hackl, arXiv:1712.02724

[33] M. D. Watson, T. K. Kim, A. A. Haghighirad, S. F. Blake, N. R. Davies, M. Hoesch, T. Wolf, and A. I. Coldea, Phys. Rev. B 92, 121108(R) (2015).

[34] I. Leonov, V. I. Anisimov, and D. Vollhardt, Phys. Rev. B 91, 195115 (2015); I. Leonov, Phys. Rev. B 92, 085142 (2015); I. Leonov, L. Pourovskii, A. Georges, and I. A. Abrikosov, Phys. Rev. B 94, 155135 (2016).

[35] Y. S. Kushnirenko, A. A. Kordyuk, A. V. Fedorov, E. Haubold, T. Wolf, B. Büchner, and S. V. Borisenko, Phys. Rev. B 96, 100504(R) (2017). 
[36] F. Birch, Phys. Rev. 71, 809 (1947).

[37] S. Baroni, S. de Gironcoli, A. Dal Corso, and P. Giannozzi, Rev. Mod. Phys. 73, 515 (2001); P. Giannozzi, S. Baroni, N. Bonini, M. Calandra, R. Car et al., J. Phys.:Condens. Matter 21, 395502 (2009).

[38] V. I. Anisimov, D. E. Kondakov, A. V. Kozhevnikov, I. A. Nekrasov, Z. V. Pchelkina, J. W. Allen, S.-K. Mo, H.-D. Kim, P. Metcalf, S. Suga, A. Sekiyama, G. Keller, I. Leonov, X. Ren, and D. Vollhardt, Phys. Rev. B 71, 125119 (2005); Dm. Korotin, A. V. Kozhevnikov, S. L. Skornyakov, I. Leonov, N. Binggeli, V. I. Anisimov, and G. Trimarchi, Eur. Phys. J. B 65, 91-98 (2008); G. Trimarchi, I. Leonov, N. Binggeli, Dm. Korotin, and V. I. Anisimov, J. Phys.: Condens. Matter 20, 135227 (2008).

[39] P. Werner, A. Comanac, L. de Medici, M. Troyer, and A. J. Millis, Phys. Rev. Lett. 97, 076405 (2006); E. Gull, A. J. Millis, A. I. Lichtenstein, A. N. Rubtsov, M. Troyer, and P. Werner, Rev. Mod. Phys. 83, 349 (2011).

[40] K. Haule, J. H. Shim, and G. Kotliar, Phys. Rev. Lett. 100, 226402 (2008); V. I. Anisimov, D. M. Korotin, M. A. Korotin, A. V. Kozhevnikov, J. Kuneš, A. O. Shorikov, S. L. Skornyakov, and S. V. Streltsov, J. Phys. Condens. Matter 21, 075602 (2009); M. Aichhorn, L. Pourovskii, V. Vildosola, M. Ferrero, O. Parcollet, T. Miyake, A. Georges, and S. Biermann, Phys. Rev. B 80, 085101 (2009); S. L. Skornyakov, A. V. Efremov, N. A. Skorikov, M. A. Korotin, Yu. A. Izyumov, V. I. Anisimov, A. V. Kozhevnikov, and D. Vollhardt, Phys. Rev. B 80, 092501 (2009); S. L. Skornyakov, A. A. Katanin, and V. I. Anisimov, Phys. Rev. Lett. 106, 047007 (2011);
Z. P. Yin, K. Haule, and G. Kotliar, Nat. Mater. 10, 932 (2011); Z. P. Yin, K. Haule, and G. Kotliar, Nat. Phys. 7, 294 (2011); M. Aichhorn, L. Pourovskii, and A. Georges, Phys. Rev. B 84, 054529 (2011); J. M. Tomczak, M. van Schilfgaarde, and G. Kotliar, Phys. Rev. Lett. 109, 237010 (2012); Z. P. Yin, K. Haule, and G. Kotliar, Phys. Rev. B 86, 195141 (2012); P. Werner, M. Casula, T. Miyake, F. Aryasetiawan, A. J. Millis, and S. Biermann, Nat. Phys. 8, 331 (2012); A. Georges, L. de Medici, and J. Mravlje, Annu. Rev. Condens. Matter Phys. 4, 137 (2013); M. Hirayama, T. Miyake, and M. Imada, Phys. Rev. B 87, 195144 (2013); C. Zhang, L. W. Harriger, Z. Yin, W. Lv, M. Wang, G. Tan, Y. Song, D.L. Abernathy, W. Tian, T. Egami, K. Haule, G. Kotliar, and P. Dai, Phys. Rev. Lett. 112, 217202 (2014); A. van Roekeghem, L. Vaugier, H. Jiang, and S. Biermann, Phys. Rev. B 94, 125147 (2016).

[41] H. J. Vidberg and J. W. Serene, J. Low Temp. Phys. 29, 179 (1977).

[42] R. S. Kumar, Yi Zhang, S. Sinogeikin, Y. Xiao, S. Kumar, P. Chow, A. L. Cornelius and C. Chen, J. Phys. Chem. B 114, 12597 (2010).

[43] F. Ricci and G. Profeta, Phys. Rev. B 87, 184105 (2013).

[44] J. Miao, X. H. Niu, D. F. Xu, Q. Yao, Q. Y. Chen, T. P. Ying, S. Y. Li, Y. F. Fang, J. C. Zhang, S. Ideta, K. Tanaka, B. P. Xie, D. L. Feng, and F. Chen, Phys. Rev. B 95, 205127 (2017).

[45] Y. Yang, W.-S. Wang, H.-Y. Lu, Y.-Y. Xiang, and Q.-H. Wang, Phys. Rev. B 93, 104514 (2016). 